\title{
Solvent-Free MALDI-MS: Developmental Improvements in the Reliability and the Potential of MALDI in the Analysis of Synthetic Polymers and Giant Organic Molecules
}

\author{
S. Trimpin \\ Oregon Health and Science University, Portland, Oregon, USA \\ S. Keune \\ Goldschmidt GMBH, Essen, Germany
}

\author{
H. J. Räder and K. Müllen \\ Max-Planck Institute for Polymer Research, Mainz, Germany
}

\begin{abstract}
A dry sample preparation strategy was previously established as a new method for matrix assisted laser desorption/ionization mass spectrometry (MALDI-MS), so-called solvent-free MALDI-MS. In this contribution, we examine systems that have been shown problematic with conventional solvent-based MALDI approaches. Problems frequently encountered are solubility, miscibility, and segregation effects during crystallization as a result of unfavorable analyte and matrix polarities. In all cases studied, solvent-free MALDI-MS simplified the measurement and improved the analysis. Solvent-free MALDI-MS enables more reliable results in well-known problematic systems such as polydimethylsiloxane with its segregation effects. However, even in highly compatible analyte/matrix systems such as polystyrene and dithranol, there were undesirable suppression effects when employing THF as solvent. Generally, the solvent-free method allows for more homogeneous analyte/matrix mixtures as well as higher shot-to-shot and sample-to-sample reproducibility. As a result, less laser power has to be applied, which yields milder MALDI conditions, reduced background signals, and provides better resolution of the analyte signals. Solvent-free MALDI-MS proved valuable for the characterization of nanosized material, e.g., fullereno-based structures, which indicated having an increased fragmentation-susceptibility. New analyte/matrix combinations (e.g., polyvinylpyrrolidone/dithranol) are accessible independent of solubility and compatibility in common solvents. An improved quantitation potential is recognized (e.g., insoluble polycyclic aromatic hydrocarbon against soluble dendrite precursor). The rapid and easy measurement of industrial products demonstrates the solvent-free method capable for improved throughput analysis of a variety of compounds (e.g., poly(butylmethacrylate) diol) in routine industrial analysis. Hence, this new MALDI method leads to qualitative and quantitative improvements, making it a powerful tool for analytical purposes, which may also prove to be valuable in future automation attempts. (J Am Soc Mass Spectrom 2006, 17, 661-671) (c) 2006 American Society for Mass Spectrometry
\end{abstract}

$\mathrm{M}$ atrix assisted laser desorption/ionization mass spectrometry (MALDI-MS) has been extensively applied to the characterization of soluble macromolecules $[1,2]$ and has even been used to detect intact soluble molecules with masses higher than one million $\mathrm{Da}$ $[3,4]$. The common understanding of the matrix assistance in MALDI-MS assumes a preorganized system where the

Published online March 15, 2006

Address reprint requests to Dr. S. Trimpin, OHSU/CROET, 3181 SW Sam Jackson Park Rd., Portland, OR 97239, USA. E-mail: trimpins@ohsu.edu analyte is homogeneously embedded in a matrix, allowing a "soft" desorption/ionization process [5, 6]. Hence, in MALDI-MS, sample preparation is one of the key factors that greatly influence the success and the quality of the mass spectrometric analysis. Since there are too many contributing parameters in solvent-based sample preparation for MALDI-MS (solvent-based MALDI-MS), there is a need for a more general method that is easily applicable and has fewer critical parameters. One critical parameter is the solvent that is necessary for homogenization of the sample and the matrix in the liquid phase and is required 
to be removed before the measurement. Three methods were developed independently with the goal to characterize solubility-restricted compounds and thus avoid the use of solvent during sample preparation. Solubilityrestricted polyamides $(<5000 \mathrm{Da})$ were characterized following a mechanical mixing of analyte and matrix by mortar and pestle and pressed pellet application of the obtained powder (solvent-free pressed pellet method) [7]. A matrix vapor deposition method was used to characterize soluble poly(ethylene glycol) (PEG, $6000 \mathrm{Da})$ [8]. Mechanical mixing of sample and matrix with a ball mill and dry transfer of the resulting powder onto the surface of the MALDI sample holder with a spatula (solvent-free method) allowed the characterization of insoluble giant polycyclic aromatic hydrocarbons (PAHs, >5700 Da) [9, $10,11]$, polyfluorene $(<10,000 \mathrm{Da})$ [12], and polydithiathianthrene $(<4000 \mathrm{Da})$ [13]. For this solventless approach, a true matrix assistance was found for UV-absorbing compounds (e.g., labile side-chain protected synthetic peptides [14] and pigments [15]), and high molecular weight polymers (e.g., poly(methyl methacrylate) and polystyrene) [15], and justified this method as a true MALDI sample preparation method. Subsequently, this solvent-free MALDI method allowed the analysis of pyrolysis products [16], carbonaceous pitches [17], and polyaniline oligomers [18]. The applied mechanical mixing methods, such as ball mill or mortar and pestle treatment, do not appear to have much influence on the success of the solvent-free MALDI analysis, however the transfer method to the MALDI sample holder does which includes improved sensitivity, higher mass resolution, and less restrictive molar analyte:matrix ratios when employing the loose powder transfer [15]. In addition, the solvent-free MALDI results indicate that there is no requirement for a highly crystalline analyte:matrix preorganization [15] and, hence, the original model of an analyte embedded in the matrix crystal seems to be a misconception $[19,20]$.

In an effort to further characterize solvent-free MALDIMS, a series of compounds (Scheme 1) and compound mixtures that in one way or another are problematic with solvent-based MALDI-MS were compared to results obtained with those from solvent-free MALDI-MS. These systems represent problems associated with segregation due to crystallization effects; analyte, matrix and solvent incompatibility issues; the influence of appropriate MALDI conditions in the solvent-free versus solventbased MALDI analysis of a compound containing a UV adsorbing moiety; suppression and solubility problems in complex mixture analysis; as well as unusual matrix solvent driven problems in highly compatible systems. High throughput issues are also addressed.

\section{Experimental}

\section{Materials}

Poly(methyl methacrylate) (PMMA, 2000 and 7100 Da) and poly(ethylene glycol) (PEG, $2000 \mathrm{Da}$ ) were obtained from Polymer Standards (Mainz, Germany). PMMA (2010 Da) was purchased from Macherey-Nagel (Düren, Germany). Poly(vinylpyrrolidone) (PVP, 2500 and 10,000 Da) was purchased from Polysciences Europe (Eppelheim, Germany). Industrial products such as poly(dimethylsiloxane) (PDMS, viscosity 450 cSt.), poly(butylmethacrylate) diole (BD-1000), and partial glycerides were provided by Goldschmidt GMBH (Essen, Germany). Various polystyrene (PS, 1600 to 2300 Da) samples were gifts from PSS (Mainz, Germany). The dendrite precursor $\mathrm{C}_{132} \mathrm{H}_{90}$ was synthesized as described elsewhere [21]. The polycyclic aromatic hydrocarbon (PAH) $\mathrm{C}_{132} \mathrm{H}_{34}$ was synthesized by cyclodehydrogenation from dendritic $\mathrm{C}_{132} \mathrm{H}_{90}$ in $\mathrm{CH}_{2} \mathrm{Cl}_{2}$ [22]. Fullerenodendron was synthesized as described elsewhere [23]. The major analyte structures are depicted in Scheme 1. 1,8,9-Trihydroxyanthracene (dithranol), 3 $\beta$-indole acrylic acid (IAA), 7,7,8,8-tetracyanoquinodimethane (TCNQ), 2-(4-hydroxyphenylazo)-benzoic acid (HABA), 9-nitroanthracene (NA), 2,5-dihydroxybenzoeic acid (DHB), and sinapinic acid (SA) were obtained from Aldrich (Steinheim, Germany). $\alpha$-Cyanohydroxycinnamic acid (HCCA) was obtained from Sigma (St. Louis, MO). All-trans retinoic acid (VitA) was obtained from Fluka (Buchs, Switzerland). Silver trifluoroacetate, lithium trifluoroacetate, sodium trifluoroacetate, and potassium trifluoroacetate were purchased from Aldrich (Steinheim, Germany) and used without further purification. Silver powder $(<60 \mu 99.9 \%)$, was obtained from $\mathrm{ABCR}$ (Karlsruhe, Germany). Tetrahydrofuran (THF, 99.8\%) was obtained from Fluka (Buchs, Switzerland).

\section{MALDI Instrumentation and Calibration}

Mass spectra were recorded using a Bruker Reflex II MALDI-TOF mass spectrometer (Bremen, Germany) equipped with a $\mathrm{N}_{2}$-laser $(\lambda=337 \mathrm{~nm})$ operating at a pulse rate of $3 \mathrm{~Hz}$. The ions were accelerated with pulsed ion extraction (PIE design from Bruker) with a voltage of $20 \mathrm{kV}$. The analyzer was operated in reflection mode and the ions were detected using a micro channel plate detector. Calibration was carried out before each measurement using a 2000 Da PS standard. PS was dissolved in THF together with silver salt (1:500:10 $\mathrm{M}$ analyte:matrix:silver trifluoroacetate) and a multi point calibration of the isotopically resolved PS oligomers was performed. To insure identical experimental conditions while comparing solvent-free and solvent-based MALDI-MS, the acceleration voltage and laser power were held constant.

Industrial laboratory. The mass spectra were recorded using a VISION 2000 (Finnigan MAT, Bremen, Germany) equipped with a $\mathrm{N}_{2}$-laser $(\lambda=337 \mathrm{~nm})$ operating at a pulse rate of $3 \mathrm{~Hz}$. The ions were accelerated by a voltage of $6 \mathrm{kV}$. The analyzer was operated in the reflection mode. The ions were detected using a secondary electron multiplier detector and a post acceleration voltage of $20 \mathrm{kV}$. Calibration was carried out with 


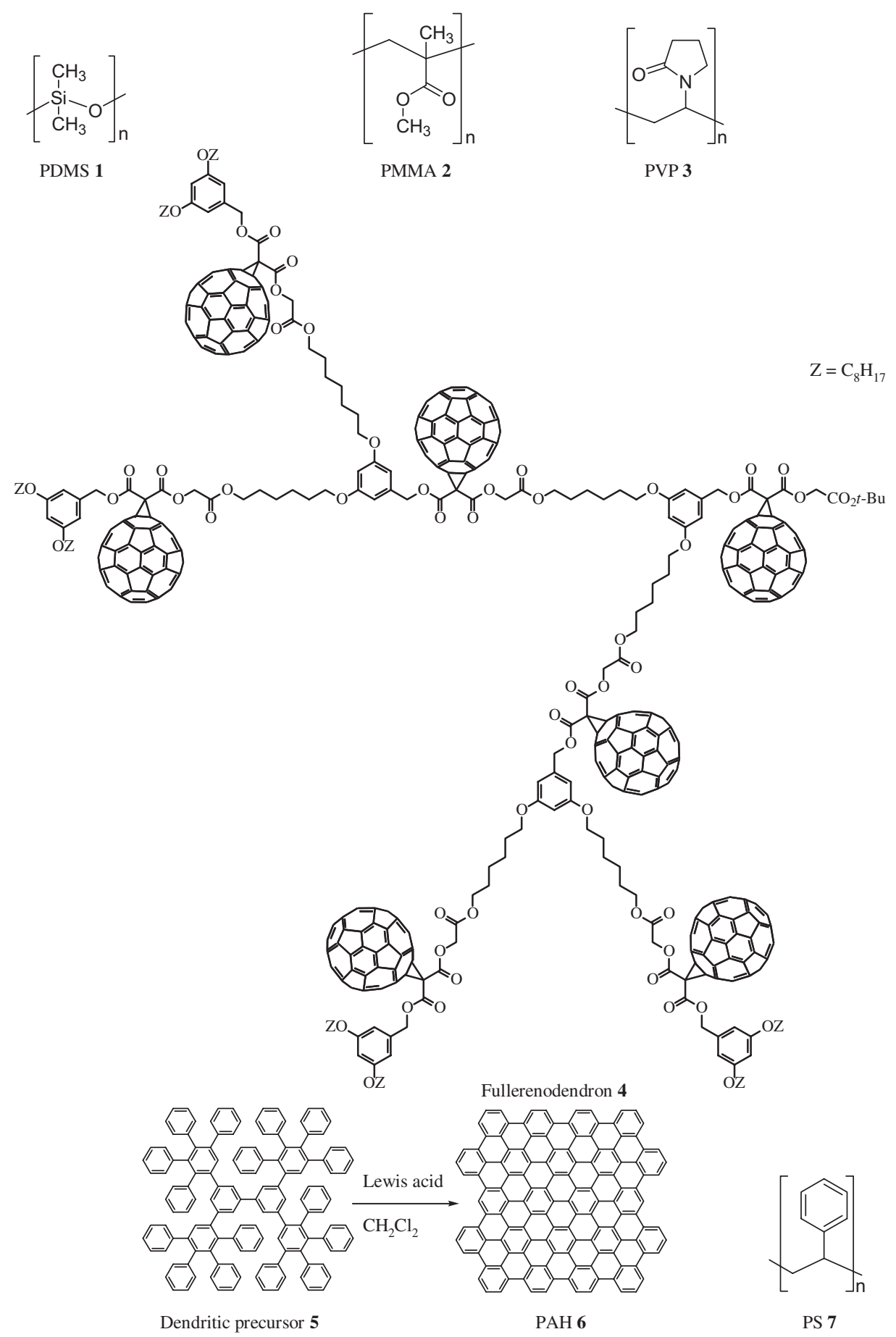

Scheme 1. Structures of the analytes 
similar molecular weight standards, e.g., PDMS was calibrated using 7100 Da PMMA, and BD-1000 was calibrated using 2010 Da PMMA.

\section{Solvent-free and Conventional Solvent-Based MALDI Sample and Target Preparation}

General procedure. The mixing of analyte and matrix powder was accomplished using a MM2000 ball mill from F. Kurt Retsch GmbH and Co. KG (Haan, Germany). Mortar and pestle treatment was applied in the industrial laboratory of Goldschmidt GMBH. For the ball mill application, the general solvent-free protocol [15] of 1:50 to 1:5000 analyte:matrix molar ratios was used. A direct comparison of solvent-free and solventbased MALDI-MS was obtained by dividing the solvent-free prepared analyte:matrix powder mixtures. One part of the analyte:matrix mixture was applied to the target as a fine powder to produce a thin coverage. The other part was completely dissolved in an appropriate solvent (e.g., THF, dichloromethane) and applied to the sample holder as a solution. After rapid evaporation of the solvent, both samples were measured with exactly the same experimental parameters.

Detailed preparation conditions. DHB (previously ground in a ball mill) and silver metal were mixed in a 2.5:1 weight ratio. The oily PDMS 1, $450 \mathrm{cSt}$ (correlating to $\sim 20,000 \mathrm{Da}$ ), was mixed with the premixed DHB: silver powder in a ratio of 1:15 via mortar and pestle treatment. PMMA 2, (2000 Da):IAA:Li salt, and PVP 3, (2500 Da):dithranol:Na salt, were each prepared in molar ratios of 1:500:10 and 1:5000:10. PVP 3, (10,000 Da) was investigated using a 1:50 molar analyte:matrix (IAA, DHB, HABA, dithranol, HCCA, NA, SA, VitA, and TCNQ) ratio. The solvent-free MALDI powder mixture of fullerenodendron 4 was prepared in a molar analyte:matrix ratio of 1:500 using TCNQ and 1:500:10 or 1:500:(no salt) for dithranol with sodium, potassium, and silver metal salts. Dendrite precursor $\mathrm{C}_{132} \mathrm{H}_{90} 5$ (white powder) and the $\mathrm{PAH} \mathrm{C}_{132} \mathrm{H}_{34} 6$ product compound (black powder) were first characterized independently. The dendrimer precursor $\mathrm{C}_{132} \mathrm{H}_{90} 5$ was mass spectrometrically pure, the $\mathrm{PAH} \mathrm{C}_{132} \mathrm{H}_{34} 6$ showed slight signals that account for incompletely cyclized side-products. From our previous studies of these compounds [9], we were able to evaluate these as minor impurities. Hence, both samples were sufficiently pure for the model MALDI investigations. The dendrite precursor $\mathbf{5}$ and the PAH product $\mathbf{6}$ were then mixed in a molar precursor:product ratio of 10:90. The solvent-free powder mixture was prepared in a molar ratio of 1:500 using TCNQ as matrix, yielding a black powder. For comparison to solvent-based MALDI-MS, this solvent-free MALDI sample was dissolved in dichloromethane. PS 7, (1600 to $2300 \mathrm{Da})$ :dithranol:silver salt was prepared for a matrix dilution experiment in ratios of 1:50:10, 1:500:10, 1:5000:10 for solvent-free and solvent-based MALDI-MS. The latter samples were prepared by dissolving the obtained powder mixture in either THF or $\mathrm{CH}_{2} \mathrm{Cl}_{2}$, as well as by directly mixing the THF dissolved analyte, matrix and salt together. Important polymers originating from industrial production such as poly(alkyleneglycol), polysiloxane copolymers, organo-modified siloxanes, functional acrylic polymers, and nonionic surfactants (e.g., ethoxylated fatty alcohols, poly(hydroxyether)s, and PBMA-PEGblockcopolymers) were investigated for routine analysis in the industrial laboratory of Goldschmidt GMBH. The analytes were mixed with a premixed matrix (DHB: $\mathrm{NaCl} 10: 1)$ in an approximate ratio between 1:10 to 1:15. Careful weighing was not employed. It was necessary to change the sample preparation conditions in two cases: PDMS was cationized with silver metal and the hydroxyl-functionalized siloxanes with $\mathrm{AgNO}_{3}$.

\section{Results and Discussion}

\section{Prevention of Segregation}

Solvent-free MALDI-MS appears to produce analyte: matrix mixtures with sufficiently high homogeneity [15] in comparison to solvent-based MALDI-MS that we were encouraged to apply this new method in specific cases where segregation of matrix and analyte is problematic [24, 25]. To understand the generality of segregation problems, one has to keep in mind that (re)crystallization is commonly used as a purification step in synthetic chemistry. Any crystallization procedure involves separation phenomena of two or more compounds in differing degrees. Intrinsically, any solventbased MALDI sample preparation suffers a lack of homogeneity caused by solvent evaporation during the crystallization step. To investigate possible improvements in MALDI-MS utilizing the solvent-free sample preparation method, results from solvent-free and solvent-based MALDI-MS of poly(dimethylsiloxane)

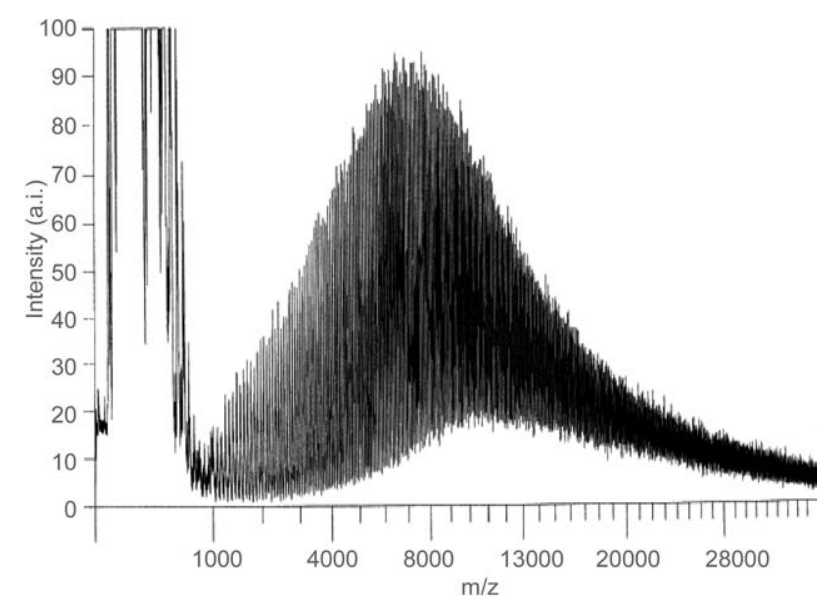

Figure 1. Solvent-free MALDI mass spectrum of industrial PDMS 1 (450 cSt.). The mass spectrum was recorded using a VISION 2000 (Finnigan MAT) instrument equipped with a $\mathrm{N}_{2^{-}}$ laser. 
(PDMS 1, Scheme 1), a well-known candidate for segregation effects, were compared [25]. A mass spectrum was conveniently obtained by solvent-free MALDI-MS (Figure 1) from a sample originating directly from industrial production utilizing the mortar and pestle treatment. The oily character of the PDMS 1 still allowed for sufficient homogenization with the matrix. Mass spectra were acquired with a high shot-to-shot reproducibility. The measured molecular weight distribution is in good agreement with values from viscometry [26]. In spite of the broad molecular weight distribution (mass range from 1000 to $28,000 \mathrm{Da}$ ), the mass spectrum showed a good signal-to-noise ratio and indicated resolved signals with repeating units of $74 \mathrm{Da}$ up to $n=450$. In contrast, solvent-based MALDI-MS was unable to acquire a mass spectrum of PDMS 1 in this mass range. The solvent-based prepared sample on the MALDI sample holder appears inhomogeneous after evaporation of the solvent and optimization trials were poor. These results suggest that solvent-based MALDI methods are difficult for this analyte due to strong segregation phenomena, which is in agreement with previous observations [25]. Solvent-based MALDI measurements of the molecular weight distribution of PDMS appear to give errors even for low molecular weight samples (<6000 Da) [25].

The polarity differences between analytes, matrices, and solvents, which cause separation effects during crystallization in solvent-based methods, are problematic as well as a source of trace impurities such as contamination by a binary solvent. Different sources can account for the origin of binary solvents. For example, traces of water are described as impurities in PMMA solutions, creating inhomogeneity and significant mass discrimination in solvent-based MALDI-MS [27]. These kinds of disadvantages were overcome by evaporation of the solvent of any problematic analyte to complete dryness and subsequent solvent-free MALDI-MS.

These investigations showed that errors in the MALDI analysis caused by segregation phenomena, previously attributed to the PDMS [25], are overcome by application of the solvent-free method. The advantage of going from a three-component (analyte, matrix, and solvent) to a two-component (analyte and matrix) system provides fewer combinations that need to be attempted. Now, disregarding optimization trials, solvent-based MALDI uses less material than the solvent-free approach, yet this is not so significant because sample requirements of less than $0.1 \mathrm{mg}$ represent still very small amounts and are not a real limitation in practice. Most polymeric samples are typically available in sufficient quantities compared to biologically-relevant amounts; however, pmol amounts are now easily accessible for MS and MS/MS measurements employing the solvent-free mini-ball mill approach $[28,29]$.

\section{Freedom in Analyte: Matrix Combinations}

The strongest correlation to the quality of mass spectra utilizing solvent-free MALDI-MS is given by the brittleness of the analyte and the matrix themselves and their ability to form homogenous sample mixtures upon the application of the external mechanical forces [15]. In solvent-based MALDI-MS some analyte:matrix combinations cannot be applied due to solvent incompatibility. In the most fortuitous situation, a solvent may be found that would allow suspension of the analyte and yield a sufficient homogeneity for matrix-assisted desorption/ionization. The aspect of solubility is important since reagents and products in a range of reactions switch from solubility in an aqueous to solubility in an organic phase (or vice versa). This precludes following the course of the reaction since the mass spectrometric result strongly depends on the applied solvent and its applicable matrix when utilizing solvent-based methods. For example, using an organic solvent would preferentially overestimate compounds that are soluble in the organic phase. On the other hand, the results of the PDMS investigations above infer that solvent-free MALDI-MS generally bears fewer compatibility restrictions. Various new analyte:matrix combinations that cannot be applied in solvent-based MALDI-MS due to the incompatibility of aqueous and organic solvent systems were explored. These beneficial new combinations were explored with the polymer standard (Scheme 1) poly(methylmethacrylate) (PMMA 2) and poly(vinylpyrrolidone) (PVP 3). The analyte PMMA 2 requires organic solvents whereas PVP 3 only swells in organic solvents and, hence, necessitates an aqueous solvent [30]. The matrix $3 \beta$-indole acrylic acid (IAA) is soluble in methanol, whereas 1,8,9-trihydroxyanthracene (dithranol) is soluble in less polar organic solvents (e.g., THF). To demonstrate the new and advantageous combinations, solvent-free MALDI-MS was utilized for unusual analyte: matrix combinations such as PMMA 2:IAA and PVP 3:dithranol in molar analyte:matrix ratios of 1:500. These analytes were characterized by mass spectra with high quality, strong signal intensities and signal-to-noise ratios in both cases PMMA 2:IAA (Figure 1a in the Supplementary Material section, which can be found in the electronic version of this article) and PVP 3:dithranol (Figure 1b also in the Supplementary Material section).

Possible effects on the molar ratios for the new analyte:matrix combinations utilizing solvent-free MALDI-MS were also investigated. For solvent-based MALDI-MS, an optimal molar analyte:matrix ratio of 1:500 is often found for polymeric analytes and was verified for solvent-free MALDI-MS in an earlier investigation [15]. In the present investigations of "incompatible" analyte:matrix combinations, the optimal molar ratios were found to be around 1:500 but were not restrictive, and good results were obtained also in the molar range 1:50 to 1:5000 (data not shown). This is in accord with previous work in which solvent-free MALDI analysis also showed a flexible range in appli- 
cable molar analyte:matrix ratios for compatible analyte:matrix combinations [15].

As an application of these new beneficial combinations of analytes and matrices via solvent-free MALDIMS, higher molecular weight PVP 3 (10,000 Da with high polydispersity) was investigated. The obtained mass spectra (data not shown) unambiguously indicated matrix assistance for all the matrices used (IAA, DHB, HABA, dithranol, HCCA, NA, SA, VitA, TCNQ). As would be expected, the quality of the obtained spectra using identical experimental conditions but various matrices differed, but all the results confirm that this technique allows hitherto unknown analyte: matrix combinations.

\section{Influence of the Appropriate MALDI Conditions in the Analysis of a Fullerenodendron}

Fullerenes have absorption at the laser wavelength (337 $\mathrm{nm}$ ) and are used directly and without the addition of a matrix as a calibration standard for MALDI analysis using a two-point calibration of $\mathrm{C}_{60}, \mathrm{~m} / \mathrm{z} 720 \mathrm{Da}$ and $\mathrm{C}_{70}, \mathrm{~m} / \mathrm{z} 840$ $\mathrm{Da}$. Their ionization mechanism is through radical cation formation. Fullerene-containing compounds are problematic in MALDI analysis due to the absorption tendency of the fullerene constituents at the laser wavelength, which unavoidably causes direct excitation by laser irradiation. Hence, the MALDI process of this type of analyte is complicated by an imposition of matrix assisted excitation of the analyte by the applied matrix as well as direct laser desorption/ionization of the analyte. Consequently, an increased fragmentation tendency can be expected in the MALDI analysis of fullerene-containing analytes. The focus of these investigations is on the importance of applying appropriate MALDI conditions to a difficult, fragmentation-susceptible high molecular weight fullerenodendron 4 (Scheme 1), in which the fullerenes are located on every side branch of the dendron. Fullerenodendron was chosen as an example for the aforementioned reasons and is additionally problematic due to the various carbon-heteroatom bonds that show a higher cleavage tendency than other bonds in dendrimers [31]. Fullerenodendron was previously characterized by optimized ES-MS analysis [30], where it was shown that the fullerene-constituents are reduced in the ES source. Hence, 4 was detected in various different radical anion charge states from pentanion to heptanion. Generating radical cations was also possible. The characterization of 4 was not possible by solvent-based MALDI-MS. Solvent-free MALDI-MS allows for a soft ionization process $[12,14,15,20]$, as does the matrix TCNQ $[9,10]$, effectively reducing the required threshold laser power for a successful desorption/ionization processes in the MALDI analysis and can therefore lower the fragmentation tendencies of analytes. Solvent-free MALDI analysis allowed for a straightforward characterization of 4 when applying TCNQ as matrix (Figure 2a). As expected, the ionization was through radical cation formation. A frag-

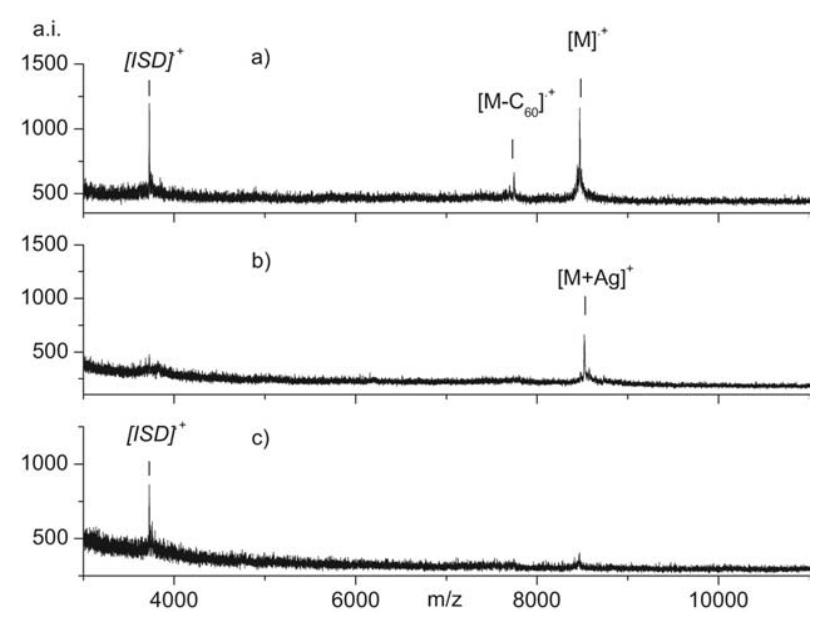

Figure 2. Solvent-free MALDI mass spectra of the fullerenodendron 4 as a function of the sample preparation conditions and their preferred ionizations: (a) 1:500 4:TCNQ; (b) 1:500:10 4:dithranol: silver salt; (c) 1:500 4:dithranol.

ment ion of $\mathrm{M}-\mathrm{C}_{60}$ was identified by its mass difference. In the lower mass region there is an intense signal of insource decay (ISD) (Figure 2a), which increases with increasing applied laser power (data not shown). This sensitivity to the applied laser power suggests that this signal also originates from fragmentation. Subsequent PSD-fragmentation studies exclude metastable decay, hence, the fragmentation must occur in the MALDI source analogously to processes in the ES source when the characterization of 4 was performed utilizing ES-MS [32].

Variations of the sample preparation conditions (e.g., dithranol and sodium, potassium, or silver salt, Figure $2 b$ ) lead to mass spectra with good quality even though higher laser power needed to be applied for the desorption/ionization process of this fragmentation-susceptible analyte. This result seems, at first, contradictive. When 4 was investigated with dithranol, in the absence of an added metal salt (Figure 2c), the parent ion was barely detected, but the signal intensity of the ISD-fragment ion was greatly increased. Overall, in the mass spectra the signal-to-noise-ratio was higher when TCNQ was applied as the matrix (Figure 2a) than when the matrix dithranol was used with (Figure 2b) or without (Figure 2c) the addition of a metal salt. Hence, TCNQ conditions that favor the radical cation formation and, therefore, the preferred ionization of the fullerene constituents of 4 necessitate less laser power in the MALDI measurement and lead to overall higher signal intensities of both the parent ion and the fragment ions (Figure 2a). We conclude that the different ionizations of 4 dictate the stability and, hence, the fragmentation tendency of this organic macromolecule: metal attachment, which leads to a pseudomolecular-ion formation, stabilizes the gas-phase ions of 4; photoionization, which leads to radical cation formation, enhances the fragmentation of 4 . Solvent-free MALDI-MS allowed for lowering the threshold laser power so that this fragmentation-labile, giant organic molecule $(>8000 \mathrm{Da})$ was characterized for the first time by MALDI analysis. 
These investigations emphasize the importance of optimizing the sample preparation conditions as well as the ease in doing so with solvent-free MALDI-MS. This study demonstrates the capability of characterizing labile nanosized material by MALDI-MS. Nanomaterials have gained rapid attention lately [33], which is reflected in increased production numbers, environmental concerns, and the lack of available appropriate analytical methods.

\section{Improved Performance for the Characterization of Complex Compound Mixtures}

It is not unusual that in a reaction mixture the precursor is more soluble than the product; in a dissolving step, the precursor and product dissolve then intrinsically to different degrees, yielding inaccurate molar ratios of the soluble and less soluble compounds in solution and, therefore, generating false MALDI results. Such a situation was simulated by preparing a mixture of welldefined monodisperse organic compounds (Scheme 1) such as soluble dendrite precursor $\mathrm{C}_{132} \mathrm{H}_{90} 5$ (in
$\mathrm{CH}_{2} \mathrm{Cl}_{2}$ ) and insoluble, cyclized polycyclic aromatic hydrocarbon (PAH) product $\mathrm{C}_{132} \mathrm{H}_{34} 6$ (in common organic solvents) in a molar precursor:product ratio of 1:9, which was then investigated by MALDI-MS. Solvent-free MALDI-MS yielded a high quality mass spectrum of the reaction mixture (Figure $3[\mathrm{I}]$ ). The MALDI sample exhibited a high homogeneity on the MALDI plate and the mass spectra were acquired with high shot-to-shot reproducibility. Both molecules are detected as radical cations. The precursor is detected to a much higher degree (about 30-fold); a 145-fold overestimation of the precursor over the product was observed previously for $\mathrm{PAH} \mathrm{C}_{222} \mathrm{H}_{42}$ [34]. When this solvent-free MALDI sample was subsequently dissolved in dichloromethane, the crystallized sample on the MALDI plate visually appeared inhomogeneous. A mass spectrum was only obtainable at much higher laser power than when solvent-free MALDI-MS was employed. Contrary to solvent-free MALDI-MS, only the soluble dendrite precursor $\mathbf{5}$ was detected (Figure 3II). The obtained solvent-based mass spectrum
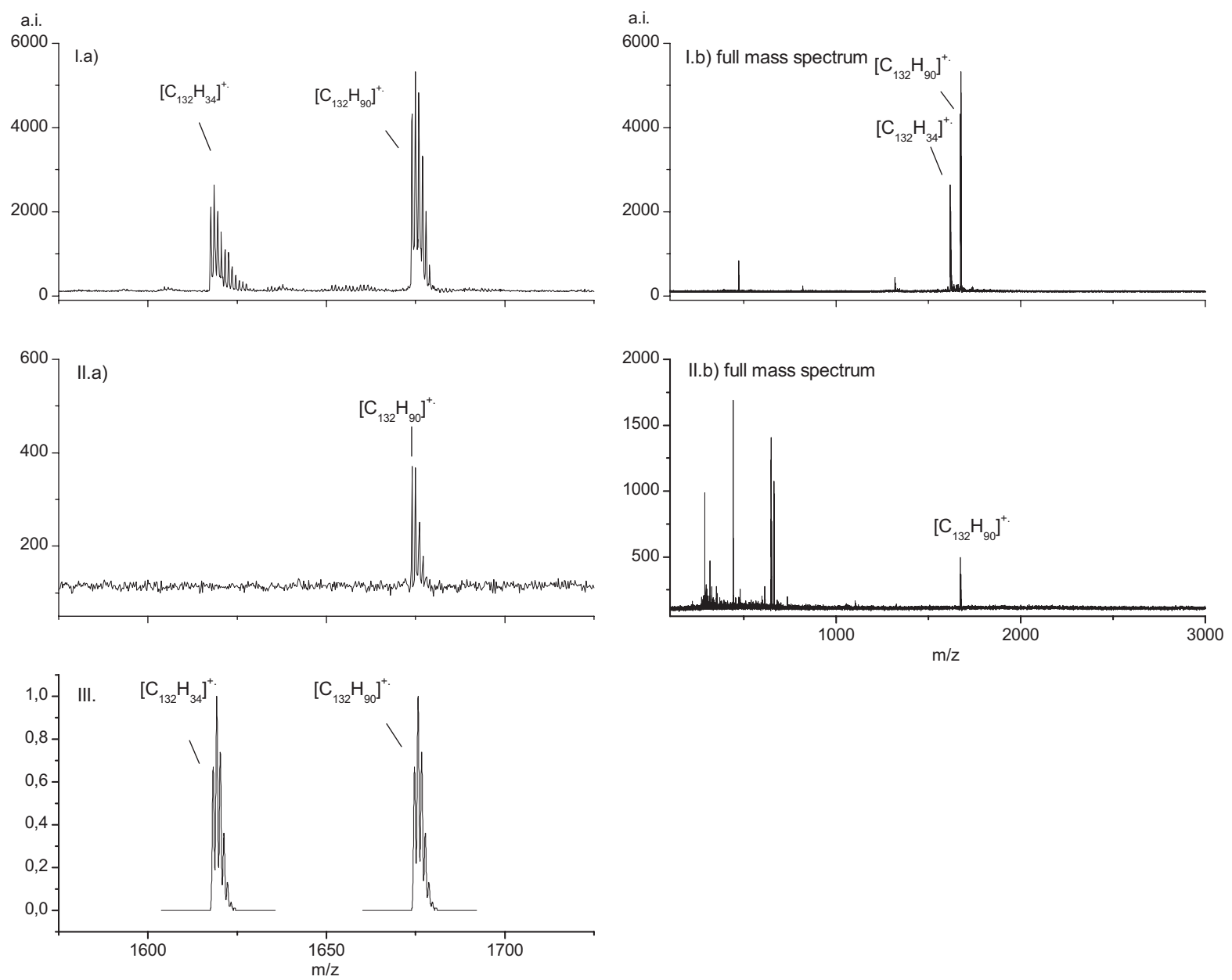

Figure 3. MALDI mass spectra of a model mixture simulating a $90 \%$ reaction yield in the synthesis the insoluble PAH product $\mathrm{C}_{132} \mathrm{H}_{34} 6$ from the dendrite precursor $\mathrm{C}_{132} \mathrm{H}_{90}$ 5: [I] Solvent-free sample preparation: (a) inset spectrum, (b) full mass spectrum; [II] conventional solvent-based sample preparation: (a) inset spectrum, (b) full mass spectrum; [III] simulated isotopic distribution of the analyte mixture calculated from the elemental compositions. 
showed, besides the complete suppression of the PAH 6, poor quality of the precursor molecule $\mathrm{C}_{132} \mathrm{H}_{34} 5$, e.g., only about $10 \%$ in intensity is observed compared to the signal intensity of 5 obtained by the solvent-free approach (Figure 3[I]a). Additionally, as shown in the full-scale mass spectra, solvent-free MALDI-MS (Figure $3[\mathrm{I}] \mathrm{b})$ produced an increased signal-to-noise ratio and less severe background signals $(<1000 \mathrm{Da})$, that are commonly observed in solvent-based MALDI-MS (Figure 3[II]b). The individual isotopic distributions were compared with the simulated isotopic distributions of the analyte mixture, which were calculated from the elemental compositions (Figure 3[III]). Precursor 5, detected via the solvent-based approach (Figure 3[II]a), did not show the expected isotopic distribution; the poor correlation is most likely the result of the higher laser power that has to be applied for successful production of molecular ions. A good agreement of the isotopic distribution is found for $\mathbf{5}$ and $\mathbf{6}$ employing the solvent-free approach (Figure 3[I]a). The high accuracy and sensitivity of solvent-free MALDI-MS for both analytes in this model compound mixture is striking and is in accord with previous investigations of, e.g., soluble polymers such as polystyrene [13]. The most severe shortcoming remains that the product 6 was not detectable with solvent-based MALDI-MS even when extremely high laser powers were applied. We rationalize the result by strong differences in the solubility of product and precursor and possible sample loss (e.g., to the wall of the tube) and segregation effects on the MALDI plate. This study emphasizes a situation of practical importance. In this case, the solvent-based MALDI result would present the wrong conclusion such as that the reaction did not occur at all even though a $90 \%$ reaction yield was obtained.

\section{Prevention of Suppression Effects in Highly Compatible Systems}

The characterization of solubility-limited compounds and/or less favorable compatible mixtures has the intrinsic advantage that the operator is aware of the challenging situation (e.g., incomplete dissolving of the analyte during sample preparation, inhomogeneities of the sample mixture on the sample holder are visually apparent) and, hence, can take appropriate precautions during sample preparation as well as when acquiring the mass spectrum. A different and somewhat more intractable situation is suppression effects in highly compatible systems because no inhomogeneities are visible. The following investigation typifies the reliability of solvent-free MALDI-MS and is discussed in greater detail to show the utility of this new MALDI method for the analysis of analytes such as polystyrene.

Routine solvent-based MALDI analysis of a polystyrene standard (PS 7, Scheme 1) using THF as solvent and dithranol as matrix, where careful weighing of the sample was omitted, showed the expected $n$-Bu- and
H-end groups as well as an additional polymer distribution (data not shown). This additional polymer distribution had a repeating unit of $104 \mathrm{Da}$. A simulation of the isotopic distribution indicated cationization with silver and excluded other common cationizing metals. The observed mass difference of $+60 \mathrm{Da}$ (or conversely $-44 \mathrm{Da}$ ) from the expected, known polymer distribution does not account for any other common end group or an analyte-matrix-adduct formation. Applying strict standard conditions (molar analyte:matrix:silver salt ratio of 1:500:10, THF as solvent) gave mass spectra with both polymer distributions. Complementary methods, including ${ }^{1} \mathrm{H}-\mathrm{NMR}$ experiments and gel-permeation chromatography (GPC) investigations, showed no PS oligomer with an additional end group and excluded high level contaminants $(>5 \%)$ in this low molecular weight PS sample. The most rational explanation of these results is a strong suppression effect of PS with the expected end group when applying solvent-based MALDI analysis. The analytical approach we used to elucidate the assumed suppression effect was a direct comparison of both MALDI methods as a function of molar analyte:matrix ratios. To ensure uniformity in sample preparation, a portion of the solvent-free analyte:matrix powder was dissolved in the appropriate solvent for use in solvent-based MALDI-MS. Thus, the methods only differed in the presence or absence of solvent.

Solvent-based MALDI-MS, using dithranol as matrix and THF as the solvent and a 1:50:10 M analyte:matrix: salt ratio, showed the expected $n$ - $\mathrm{Bu}$ - and $\mathrm{H}$-end groups (Figure 4[I]a). In a molar analyte:matrix:salt ratio of 1:500:10, an unexpected polymer distribution was observed (Figure $4[\mathrm{I}] \mathrm{b}$ ) in addition to the original polymer distribution that accounted for $n-\mathrm{Bu}^{-}$and H-end groups, whereas for a 1:5000:10 ratio only this unknown polymer distribution was detected (Figure $4[\mathrm{I}] \mathrm{c}$ ). Hence, an additional polymer distribution is obtained with increasing matrix dilution utilizing solvent-based MALDI-MS. Solvent-free MALDI experiments performed with any molar analyte:matrix ratio between 1:50:10 and 1:5000:10 (Figure 4[II]) showed in contrast only the $n$-Bu- and $\mathrm{H}$-end groups and even produced signal intensities that were equal to or much higher than with solvent-based MALDI-MS (Figure 4[I]). Repeating this comparison showed identical results and excluded errors in sample preparation. However, when analogous solvent-free and subsequent solvent-based MALDI matrix dilution experiments are performed with dichloromethane as a solvent, no additional polymer distribution is detected in any of the molar ratios between 1:50:10 and 1:5000:10. This result is shown in Figure 4[I]d for a molar ratio of 1:5000:10. Furthermore, the additional end group with +60 Da that was found for solvent-based MALDI-MS using THF (Figure 4[I]b, c) was not observed in any PS 7:IAA analyte:matrix ranging from 1:50:10 to 1:5000:10 (Figure 4[II]d, 1:5000: 10) utilizing solvent-free MALDI-MS.

A higher consistency in the results was obtained by 

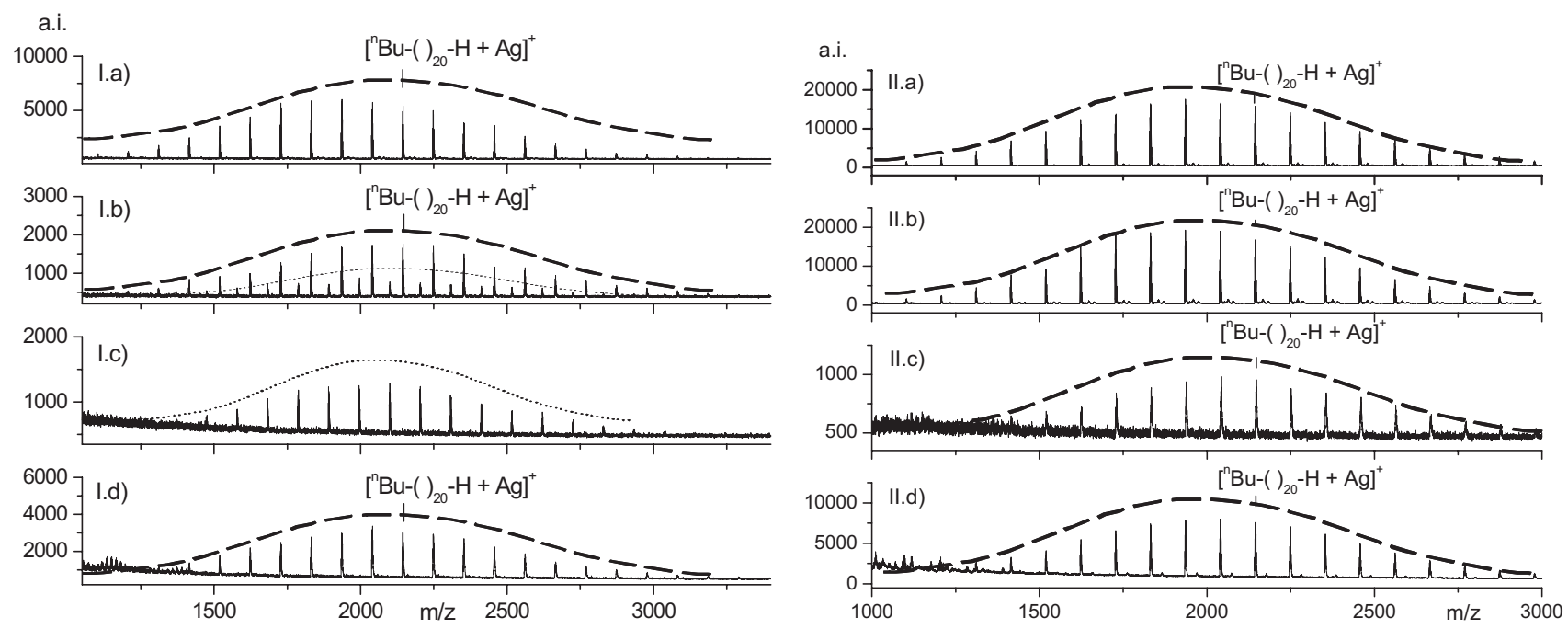

Figure 4. MALDI mass spectra of PS 7 (2000 Da) as a function of molar ratios of [I] PS:dithranol:silver salt and solvents applying conventional solvent-based MALDI-MS: (a) 1:50:10 dissolved THF; (b) 1:500:10 dissolved THF; (c) 1:5000:10 dissolved in THF; (d) 1:5000:10 dissolved in dichloromethane; [II] PS:matrix:silver salt and matrices applying solvent-free MALDI-MS: (a) 1:50:10 and dithranol; (b) 1:500:10 and dithranol; (c) 1:5000:10 and dithranol; (d) 1:5000:10 and IAA.

solvent-free MALDI-MS independent of matrix or ratios used. We assume that the misleading result of this additional polymer distribution that appears when using THF and the common and higher matrix dilutions in solvent-based MALDI-MS may be due to traces of contaminants (e.g., from traces of peroxides in THF) that are below the detection limit for ${ }^{1} \mathrm{H}-\mathrm{NMR}$ as well as for ESI- and FD-MS, MS methods that unambiguously showed the expected $n$-Bu- and H-end groups. Following this assumption, PS oligomers with similar molecular weights (1600 to $2300 \mathrm{Da}$ ) that originated from the same batch of initiator for anionic polymerization were investigated. These PS oligomers 7 show, with increasing matrix dilution, the same additional polymer distribution of +60 Da when using solvent-based MALDI-MS and THF as the solvent. PS oligomers 7 that originated from different batches of initiator showed, with high matrix dilution, only the expected $n$-Bu- and $\mathrm{H}$-end groups. Therefore, only in the batch of one initiator, a polymer side-product with an unknown end group is formed in presumably less than $5 \%$ quantities (value estimated by complementary methods). The suppression effect of the PS oligomer 7 with $n$-Bu- and H-end groups is successfully prevented utilizing solvent-free MALDI-MS. Evaluating a rough estimation of the over-representation of the unknown oligomer in the mass spectra (PS oligomer $7 n=19$ ) obtained by solvent-based MALDI-MS leads to a minimum value of $+20 \%$ for the conventional optimal molar ratio of 1:500 and $+100 \%$ for the molar ratio of $1: 5000$. Given the assumption of a maximum of $5 \%$ contamination, the solvent-free MALDI method yields an under-representation of at most $-5 \%$ independent of molar ratios. Although neither method yields a highly quantitative result, the application of solvent-free MALDI-MS, in this case, greatly reduced suppression effects. Recently [35] it was shown that a PS sample underwent oxidation at the sulfur end-group during a solvent-based sample preparation employing THF as solvent. This oxidation was not observed utilizing solvent-free MALDI-MS. Hence, the similarities in terms of analyte, matrix, and solvent, allow us to conclude that this undesired interference may be a general problem with solvent-based MALDI-MS and can be avoided employing the solvent-free method.

\section{Improved Performance for Throughput Analysis}

Typically, high throughput solvent-based MALDI analysis involves similar samples-optimal conditions for one sample are assumed to be sufficient for all samples. The robust, highly adaptable solvent-free MALDI method makes it invaluable for high-throughput investigations: the potential for the analysis of numerous compounds with various molecular weights with a minimum variation of sample preparation while maintaining sufficient quality in the mass spectra. In this routine solvent-free MALDI analysis, the following analytes were successfully characterized: poly(alkyleneglycol) (200-10,000 Da), PMMA (500-20,000 Da), polystyrene (500-10,000 Da), PDMS (700-10,000 Da), partially ethoxylated poly(hydroxyester) (up to 3000 $\mathrm{Da}$; resolution is compromised above this molecular weight) and commercially available tensides (ethoxylated with $n=2$ to 100). PDMS was cationized with silver powder and the hydroxyl-functionalized siloxanes with $\mathrm{AgNO}_{3}$, and are therefore special cases. It should be emphasized that all the other analytes were accessible with the matrix DHB, the cationizing agent $\mathrm{NaCl}$, and with the same molar ratios. The applied 
molar ratios were approximate and did not require careful weighing. This flexibility in preparation was already exploited with studies of analytes 2 and 3 . Additionally, these results were obtained in a quick, straightforward manner and without any optimization procedures.

The solvent-free approach allowed for the routine characterization of the above mentioned analytes with much improved time efficiency, and resulted in mass spectra with increased quality and a higher signal-tonoise ratio (e.g., poly(butylmethacrylate)-1000 diole; BD-1000). Due to their extreme range in polarities, thoxylated partial glycerides exhibit highly variable solubility and great difficulties in obtaining results utilizing solvent-based MALDI-MS. Nevertheless, when a compound mixture containing PEG, ethoxylated glycerine, and up to $C_{16}: C_{18}$ triglycerides was investigated using solvent-free MALDI-MS, the mass spectra obtained could be used to accurately characterize the complete compound mixture. Generalizing these results, several advantages for solvent-free MALDI-MS were found for nonpolar analytes when using the DHB matrix. In contrast, the separation effect appears to increase in solvent-based MALDI-MS when differences in the polarities of the matrix and analyte increase, resulting in insufficient mass spectral quality (e.g., BD-1000) or even prevented (e.g., PDMS) the MS analysis, presumably because of the negative mediation of the solvent.

\section{Conclusions}

The improvements in analysis gained by utilizing solvent-free MALDI-MS are striking. Future applications of solvent-free MALDI-MS may include compounds such as solid-state synthesized products (e.g., to exclude unknown solvent effects such as oxidation or degradation etc.) [36], environmental-relevant solids (e.g., contaminated soil or sludge, nanomaterials, PAHs, kerosene, and fuels etc.), as well as biological samples (e.g., solubility-limited membrane proteins) [29], all of which are sometimes only accessible by time-consuming sample pre-treatments or LD MS if at all. It is reasonable to assume that solvent-free MALDI analysis will be unable to acquire mass spectra directly of highly entangled polymers such as commercial polyester or nylon, so that further adjustments to the sample preparation might be required to access these types of highly intractable synthetic polymers in the future. Less than optimal quantitative results due to desorption/ ionization preferences, wide polydispersity, and instrumentational limitations (e.g., detector saturation) are independent of the sample preparation method. For this, focused quantitative MALDI-MS strategies must be further developed but will certainly be a difficult task, as shown previously [37]. Problems in quantitative investigations such as polydisperse samples, overrepresentation of lower molecular weight compounds, saturation of signals and detection inefficiency [38, 39], suppression effects due to preferential desorption/ionization etc. still exist, making accurate quantitation of mixtures difficult. The solvent-free MALDI method has numerous beneficial aspects as described above making it valuable for future quantitation approaches. In particular, the intrinsic over-estimation of parts of a mixture, e.g., due to preferential desorption/ionization, is more reproducible in these experiments and, therefore, can be recalculated mathematically for quantitative analysis.

Since solvent-free MALDI-MS has been shown to perform routine analysis precisely and accurately, to require less time for sample preparation, and to acquire improved mass spectra faster, it seems reasonable to propose an automated [40] solvent-free sample preparation platform for high throughput accurate mass determination (automated workstation and automated mass analysis) designed into a commercial MALDI-TOF mass spectrometer. The method and clean-up issues related to solvent-free MALDI-MS, which were considered a serious obstacle for routine and high throughput use, can be overcome using recently described solvent-free sample preparation strategies, such as vortexing [41, 42], mini-ball mill [28, 29], and in particular, on-target grinding [42] methods, which can adapt to automation as well as to downscale the required amounts of sample and matrix. In several laboratories, solvent-free MALDI is already being used in about $50 \%$ of the routine analyses performed (e.g., authors' laboratories and [41]).

\section{Acknowledgments}

The authors gratefully acknowledge M. Koerner (Goldschmidt GMBH, Essen, Germany) and A. Rouhanipour (MPI, Mainz, Germany) for support in developing this method. They thank A. Veyland (PAH sample; MPI, Mainz, Germany), J.-F. Nierengarten (dendron sample; GMO, IPCMS, Strasbourg, France), and Dr. T. Hofe (PS sample; Polymer Standards Service, Mainz, Germany) for providing samples as well as Dr. S. D. Hanton (Air Products and Chemicals, Inc., Allentown, PA) and Dr. C. N. McEwen (DuPont, Co., Wilmington, DE) in sharing their experiences with us.

\section{References}

1. Karas, M.; Bachmann, D.; Bahr, U.; Hillenkamp, F. Matrix assisted ultraviolet laser desorption of nonvolatile compounds. Int. J. Mass Spectrom. Ion Processes 1987, 78, 53-68.

2. Tanaka, K.; Waki, H.; Ido, Y.; Ákita, S.; Yoshida, Y.; Yoshida, T. Protein and polymer analyses up to $m / z 100,000$ by laser ionization time-offlight mass spectrometry. Commun. Mass Spectrom. 1988, 2, 151-153.

3. Schriemer, D. C.; Li, L. Detection of high molecular weight narrow polydisperse polymers up to 1.5 million Daltons by MALDI mass spectrometry. Anal. Chem. 1996, 68, 2721-2725.

4. Wenzel, R. J. Matter, U.; Schultheis, L.; Zenobi, R. Analysis of megadalton ions using cryodetection MALDI time-of-flight mass spectrometry. Anal. Chem. 2005, 77, 4329-4337.

5. Chan, T. W. D.; Colburn, A. W.; Derrick, P. J.; Gardiner, D. J.; Bowden, M. Suppression of matrix ions in ultraviolet laser desorption: Scanning electron microscopy and Raman spectroscopy of the solid samples. Org. Mass Spectrom. 1992, 27, 188-194.

6. Doktycz, S. J.; Savickas, P. J.; Krueger, D. A. Matrix/sample interactions in ultraviolet laser-desorption of proteins. Rapid Commun. Mass Spectrom. 1991, 5, 145-148. 
7. Skelton, R.; Dubois, F.; Zenobi, R. A MALDI sample preparation method suitable for insoluble polymers. Anal. Chem. 2000, 72, 17071710 .

8. Marie, A.; Fournier, F. Tabet, J. C. Characterization of synthetic polymers by MALDI-TOF/MS: Investigation into new methods of sample target preparation and consequence on mass spectrum finger print. Anal. Chem. 2000, 72, 5106-5114.

9. Przybilla, L. M.; Brand, J. D.; Yoshimura, K.; Räder, H. J.; Müllen, K. Characterization of large synthetic polycyclic aromatic hydrocarbons by MALDI- and LD-TOF mass spectrometry. Anal. Chem. 2000, 72, 45914597.

10. Simpson, C. D.; Mattersteig, G.; Martin, K.; Gherghel, L.; Bauer, R. E.; Räder, H. J.; Müllen, K. Nanosized molecular propellers by cyclodehydrogenation of polyphenylene dendrimers. J. Amer. Chem. Soc. 2004, 126 (10), 3139-3147.

11. Räder, H. J.; Rouhanipour, A.; Talarico, A. M.; Palermo, V.; Samori, P.; Müllen, K. Processing of giant graphene molecules by soft-landing mass spectrometry. Nature Materials accepted 2006.

12. Trimpin, S.; Grimsdale, A. C.; Räder, H. J.; Müllen, K. Characterization of an insoluble poly(9,9-diphenyl-2,7-fluorene) by solvent-free sample preparation for MALDI-TOF mass spectrometry. Anal. Chem. 2002, 74, 3777-3782.

13. Leuninger, J.; Trimpin, S.; Räder, H. J.; Müllen, K. Novel approach to ladder-type polymers: Polydithiathianthrene via the intramolecular acid-induced cyclization of methylsulfinyl-substituted poly (metaphenylene sulfide). Macromol. Chem. Phys. 2001, 202, 2832-2842.

14. Trimpin, S.; Klok, H. A.; Mayer-Posner, F. J.; Räder, H. J. A comparative study of MALDI-PSD and MALDI-CID fragmentation for structural elucidation of synthetic polypeptides. Proceedings of the 48th ASMS Conference on Mass Spectrometry and Allied Topics. Long Beach, CA, June 2000.

15. Trimpin, S.; Rouhanipour, A.; Az, R.; Räder, H. J.; Müllen, K. New aspects in matrix assisted laser desorption/ionization time-of-flight mass spectrometry: A universal solvent-free sample preparation. Rapid Commun. Mass Spectrom. 2001, 15, 1364-1373.

16. Gherghel, L.; Kübler, C.; Lieser, G.; Räder, H. J., Müllen, K. Pyrolysis in the mesophase: A chemist's approach toward preparing carbon nanoand microparticles. J. Am. Chem. Soc. 2002, 124, 13130-13138.

17. Edwards, W. F.; Jin, L. W.; Thies, M. C. MALDI-TOF mass spectrometry: Obtaining reliable mass spectra for insoluble carbonaceous pitches. Carbon 2003, 41, 2761-2768.

18. Dolan, A. R.; Wood, T. D. Analysis of polyaniline oligomers by laser desorption ionization and solventless MALDI. J. Am. Soc. Mass Spectrom. 2004, 15, 893-899.

19. Trimpin, S.; Räder, H. J.; Müllen, K. MALDI-TOF Mass SpectrometryPushing the Limits. Proceedings of the 50th ASMS Conference on Mass Spectrometry and Allied Topics Orlando, FL, June 2002.

20. Trimpin, S.; Räder, H. J.; Müllen, K. Investigations of theoretical principles for MALDI-MS derived from solvent-free sample preparation. Part I, Preorganization. Int. J. Mass Spectrom, in press.

21. Berresheim, A. J., Ph. D. Thesis, Polyphenylen-Dendrimere, Johannes Gutenberg-Universität, Mainz, 2000, p 169.

22. Morgenroth, F.; Reuther, E.; Mullen, K. Polyphenylene dendrimers: From three-dimensional to two-dimensional structures. Angew. Chem. Int. Ed. Eng. 1997, 36, 631-634

23. Felder, D.; Nierengarten, H.; Gisselbrecht, J. P.; Boudon, C.; Leize, E.; Nicoud, J. F.; Gross, M.; Van Dorsselaer, A.; Nierengarten, J. F. Fullerodendrons: Synthesis, electrochemistry, and reduction in the electrospray source for mass spectrometry analysis. New J. Chem. 2000, 9, 687-695.

24. Hanton, S. D.; Clark, P. A. C.; Owens, K. G. Investigations of matrix assisted laser desorption/ionization sample preparation by time-of- flight secondary ion mass spectrometry. J. Am. Soc. Mass Spectrom. 1999, 10, 104-111.

25. Montaudo, G.; Montaudo, M. S.; Puglisi, C.; Samperi, F. Molecular weight distribution of poly(dimethylsiloxane) by combining matrix assisted laser desorption/ionization time-of-flight mass spectrometry with gel-permeation chromatography fractionation. Rapid Commun. Mass Spectrom. 1995, 9, 1158-1163.

26. Barry, A. J. Viscometric investigation of dimethylsiloxane polymers. J. Appl. Phys. 1946, 17, 1020-1024.

27. Chen, H. R.; Guo, B. C. Use of binary solvent systems in the MALDITOF analysis of poly(methyl methacrylate). Anal. Chem. 1997, 69, 4399-4404.

28. Trimpin, S.; Deinzer, M. L. Solvent-free MALDI-MS for the analysis of biological samples via a mini-ball mill approach. J. Am. Mass Spectrom. 2005, 16, 542-547.

29. Trimpin, S.; Spencer, P. S.; Deinzer, M. L. Evaluation of solvent-free MALDI-MS for the analysis of proteins via the mini-ball mill. Mol. Cell. Proteom. 2005, 4(Suppl. 1), S311.

30. Trimpin, S.; Eichhorn, P.; Räder, H. J.; Müllen, K.; Knepper, T. P. Recalcitrance of poly(vinylpyrrolidone): Evidence through matrix assisted laser desorption-ionization time-of-flight mass spectrometry. J. Chromatogr. A 2001, 938, 67-77.

31. Trimpin, S.; Grebel-Koehler, D.; Räder, H. J.; Müllen, K. Evaluation of solvent-free matrix assisted laser desorption/ionization (MALDI) mass spectrometry for the analysis of dendrimers; unpublished.

32. Nierengarten, H.; Felder, D.; Leize, E.; Nierengarten, J.-F.; Nicoud, J.-F.; Van Dorsselaer, A.Characterization by ESMS of neutral fullerenefunctionalized dendritic branches. Proceedings of the 48th ASMS Conference on Mass Spectrometry and Allied Topics. Long Beach, CA, June 2000.

33. Colvin, V.Eco-Nano: The Impact of Engineered Nanomaterials on the Environment. Proceedings of the Society of Environmental Toxicology and Chemistry 26th Annual Meeting in North America. Baltimore, MD, November 13-17, 2005,

34. Przybilla, L. Ph.D. Thesis, Methodische Entwicklung der MALDI-TOFMassenspektrometrie für Grenzbereiche der Polymeranalytik, Johannes Gutenberg-Universität, Mainz, 2000; p 148.

35. Polce, M. J.; Wesdemiotis, C.; Kim, H.; Quirk, R. P.Investigation of MALDI sample preparation effects during end group analysis of functionalized polystyrene polymers. Proceedings of the 53rd ASMS Conference on Mass Spectrometry and Allied Topics. San Antonio, TX, June 5-9, 2005.

36. Hait, S. B.; Sivaram, S. Ester-carbonate interchange reaction in the solid state: synthesis of poly(ester-carbonate). Macromol. Chem. Phys. 1998, 199, 2689-2697.

37. Wallace, W. E.Recent advances in quantitative synthetic-polymer Mass spectrometry at NIST. Proceedings of the 52nd ASMS Conference on Mass Spectrometry and Allied Topics. Nashville, TN, May 23-27, 2004

38. McEwen, C. N.; Jackson, C.; Larsen, B. S. Instrumental effects in the analysis of polymers of wide polydispersity by MALDI mass spectrometry. Int. J. Mass Spectrom. Ion Processes 1997, 160, 387-394.

39. Byrd, H. C. M.; McEwen, C. N. The limitations of MALDI-TOF mass spectrometry in the analysis of wide polydisperse polymers. Anal. Chem. 2000, 72, 4568-4576.

40. Lake, D. A.; Johnson, M. V.; McEwen, C. N.; Larsen, B. S. Sample preparation for high throughput accurate mass analysis by matrix assisted laser desorption/ionization time-of-flight mass spectrometry. Rapid Commun. Mass Spectrom. 2000, 14, 1008-1013.

41. Hanton, S. D.; Parees, D. M. Extending dry MALDI sample preparation for polymer analytes. Proceedings of the 52nd ASMS Conference on Mass Spectrometry and Allied Topics. Nashville, TN, May 23-27, 2004.

42. Hanton, S. D.; Parees, D. M. Extending the solvent-free MALDI sample preparation method J. Am. Mass Spectrom. 2005, 16, 90-93. 